Forschende

Komplementärmedizin und

Klassische Naturheilkunde
Kongressbericht - Congress Report

Forsch Komplementärmed Klass Naturheilkd 2004;11:304-305

\section{Bundestagung 2004 der Frauenselbsthilfe nach Krebs Motto: Miteinander reden, gemeinsam handeln}

Noch nie waren so viele Menschen bei einer Bundestagung der Frauenselbsthilfe nach Krebs versammelt wie in diesem Jahr. 631 Mitglieder reisten aus allen Teilen Deutschlands an, 12 Landesverbände und fast alle 430 Gruppen waren vertreten. Dazu kamen Ehrenmitglieder, Referenten und zahlreiche Gäste. Der Saal war mit nahezu 700 Personen gefüllt.

Herausragendes Ereignis war die Verleihung des Verdienstkreuzes am Bande des Verdienstordens der Bundesrepublik Deutschland an die Bundesvorsitzende der Frauenselbsthilfe nach Krebs, Hilde Schulte. Der Ministerpräsident von Sachsen-Anhalt, Prof. Dr. Wolfgang Böhmer, führte mit sehr persönlich gehaltenen Worten die Verleihung stellvertretend für den hessischen Ministerpräsidenten durch (Frau Schulte wohnt in Hessen).

Stehende Ovationen begleiteten die Verleihung. Sie ist als Wertschätzung der ehrenamtlichen Arbeit und der Verdienste, die Hilde Schulte innerhalb und ausserhalb des Verbandes erworben hat, anzusehen. Darüber hinaus ist die Ehrung sowohl Anerkennung der individuellen Leistung jedes einzelnen Mitgliedes als auch der kollektiven Leistung des Verbandes.

Die Bedeutung der Tagung über die Grenzen des Verbandes hinaus wurde deutlich durch die Teilnahme von Persönlichkeiten aus Politik und aus der Ärzteschaft, von Kostenträgern und Mitgliedern der Deutschen Krebshilfe, der Schirmherrin und von Förderern des Verbandes. Aus dem politischen Sektor seien genannt:

- Dr. Rainer Hess, Vorsitzender des Gemeinsamen Bundesausschusses. Als wichtige Aufgabenfelder dieses seit 01.01.2004 bestehenden Gremiums nannte er das Mammographie-Screening für alle Frauen von 50 bis 69 Jahren und das Disease Management Programme (DMP) Brustkrebs. Die im vergangenen Jahr vereinbarten Regelungen und Empfehlungen würden unter Beteiligung von Patientenvertretern regelmässig aktualisiert und mögliche Verbesserungen zeitnah beschlossen.

- Helga Kühn-Mengel, Patientenbeauftragte der Bundesregierung, die sich bereits in ihrer Funktion als gesundheitspolitische Sprecherin der SPD-Fraktion für die Belange von Patientinnen und Patienten einen Namen gemacht hat. Hier sei zu den oben genannten Themen noch die Zertifizierung von Brustzentren genannt. Frau Kühn-Mengel erwähnte, dass das Motto der Tagung, «Miteinander reden, gemeinsam handeln», bereits praktiziert werde.
Für die Ärzteschaft und als Mitglied des wissenschaftlichen Beirates des offiziellen Magazins der Frauenselbsthilfe nach Krebs sprach Prof. Dr. Hans Georg Bender ein Grusswort. Er hob den bereits vor langer Zeit begonnenen Dialog und die gute Zusammenarbeit mit der Selbsthilfe hervor, zum Beispiel bei der Weiterentwicklung von Strukturen und der Sicherung von Qualität und Transparenz in der medizinischen Behandlung.

Frau Prof. Dr. Marianne Brieskorn-Zinke setzte sich mit dem Thema «Patientenbeteiligung zwischen Autorität und Autonomie» auseinander. Sie beleuchtete an drei Modellen von Arzt-Patienten-Beziehung die Möglichkeiten und Gefahren der erkämpften neuen Rolle des Patienten: 1. die traditionelle Patientenrolle und der Paternalismus des Arztes; 2. die Patientin als beteiligte Expertin oder als Koproduzentin der eigenen Gesundheit; 3. die Patientin als Partnerin im Behandlungsprozess. Sie wünschte der Frauenselbsthilfe nach Krebs, dass sie ihre Vision von einer kritischen Kraft im Gesundheitswesen behalte und sich nicht verliere in der Logik der Gesundheitspolitik und ihrer Lobbyisten.

Das fachliche Programm war ausgewogen konzipiert und seine Inhalte wurden theoretisch fundiert und weitgehend praxisnah vermittelt. In den Grussworten und Vorträgen standen Plädoyers für eine verstärkte Akzeptanz und Kompetenz der Selbsthilfe und Patientenbeteiligung sowie Aussagen über wichtige Entscheidungen künftiger Gesundheitspolitik im Mittelpunkt.

Zur Verdeutlichung seien einige Themen genannt. In seinem Vortrag «Tumornachsorge - Wandel im Denken. Rehabilitation zwischen Luxus und Versorgungsleistung» machte Prof. Dr. Hans Helge Bartsch deutlich, dass Rehabilitation durchaus kein Luxus, sondern gesetzlich verankert, sinnvoll und effektiv sei, wenn Bedarf bestehe.

Frau Prof. Dr. B. Schmalfeldt stellte anschaulich den Stellenwert neuer Diagnostik- und Therapieformen beim Zervixund Ovarialkarzinom vor. Sie gab einen ausgezeichneten Überblick über Ergebnisse und Perspektiven.

PD Dr. Stephan Schmitz beschäftigte sich mit dem für viele Betroffene emotional stark belasteten Bereich der metastasierten Erkrankung, speziell mit der palliativen Chemotherapie. Gemeinsames Ziel von Arzt und Patientin sei die Verbesserung der Lebensqualität. «Sie sind austherapiert» dürfe keinem Arzt mehr über die Lippen kommen.

An das viel diskutierte und umstrittene Thema «Hormonersatztherapie und Krebs - Nutzen und Risken» wagte sich ein kompetenter Experte, Prof. Dr. G. Emons. Viele Fragen der Teilnehmerinnen wurden durch seinen Vortrag geklärt. 
Prof. Dr. J. Beuth widmete sich dem Thema «Komplementärmedizinische Massnahmen - wissenschaftlich begründet und therapeutisch effizient». Er warnte vor selbst ernannten Experten, vor Scharlatanerie und Betrug auf Kosten von Angst und Verzweiflung.

Der Vortrag «Krebs und Sport - Bewegung tut Not» von Frau Dr. A. Reinhardt lockerte mit einer praktischen Übung die vom vielen Sitzen müden Glieder wieder auf.

Viel Beifall erntete der Beitrag «Lebenslust - Risiken und Nebenwirkungen der Gesundheit» von Dr. M. Lütz, der rhetorisch brillant und humorvoll vorgetragen wurde.

Die Ausführungen zu «Kommunikation - nicht nur mit anderen, sondern auch mit mir selbst» von G. von der Lehr mit ausgewählten Beispielen wurden als sehr hilfreich für den Umgang mit der eigenen Person und für die Gruppenarbeit empfunden.
In mehreren Beiträgen wurde ein Rückblick auf die Forschung der vergangenen Jahrzehnte gegeben und die erneute Prüfung bisheriger Hypothesen und Resultate dargestellt. Trotz aller Rückschläge und noch vorhandenen Lücken in der Krebsforschung sind deutliche Fortschritte erkennbar. In ihrer Gesamtheit erweckten diese Informationen bei den Zuhörern den Eindruck intensiver Bemühungen um das Wohlergehen von Betroffenen und hinterliessen Hoffnung und Zuversicht.

«Wissen allein führt nicht zum Ziel, helfen ist wichtig» sagte Frau Prof. Sabine Freifrau von Kleist, Vorstandsmitglied der Deutschen Krebshilfe, Vorsitzende des Dr. Mildred Scheel Kreises und langjähriges Ehrenmitglied der Frauenselbsthilfe nach Krebs in ihrem Grusswort. Helfen ist weiterhin gemeinsames Anliegen der Deutschen Krebshilfe und der Frauenselbsthilfe nach Krebs (E-mail: kontakt@frauenselbsthilfe.de).

H. Schulte, Neukirchen 